\title{
Cigarette smoking and Helicobacter pylori infection
}

\author{
Malcolm C. Bateson
}

General Hospital, Bishop Auckland, County Durham DL14 6AD, UK

\begin{abstract}
Summary: The direct urease test was used in 462 patients with normal upper digestive tracts, 108 with duodenal ulcers and 43 with gastric ulcers who attended for upper digestive endoscopy in a prospective study. There was a strong association between Helicobacter pylori infection and current cigarette smoking in patients with normal endoscopy $(49.6 \% \mathrm{vs} 35.5 \%, P<0.01)$. The associations of peptic ulcer both with $H$. pylori infection and cigarette smoking were also confirmed.

The excess of peptic ulcer disease in cigarette smokers may be explained by their increased susceptibility to $H$. pylori infection.
\end{abstract}

\section{Introduction}

Cigarette smoking is an important factor in peptic ulcer disease and dyspeptic symptoms. Since the use of tobacco impairs the immune system, ${ }^{1-4}$ it was speculated that current cigarette smokers are predisposed to Helicobacter pylori infection. This was examined in a prospective study of patients attending for endoscopy using urease testing. It is known that the link between peptic ulcer and smoking cannot be explained by changes in acid output. ${ }^{5}$

The description of the presence of the spiral bacterium $H$. pylori in the stomach as a regular associate of histological antral gastritis and peptic ulcers led to new ideas about the nature of these problems and their management. ${ }^{6-8}$

Control of $H$. pylori by the use of bismuth salts is linked with a lower rate of ulcer relapse after healing than is seen with the acid-lowering drugs. However, mere reduction in the number of organisms or conversion to dormant coccoid forms is not the prime objective of therapy, which is complete elimination of $\mathrm{H}$. pylori. This should theoretically be possible with combined treatment including antibiotics and bismuth preparations. Such regimes have been promoted as a specific radical cure for benign peptic ulcer disease. ${ }^{9,10}$

The present studies were designed to obtain more information about the prevalence of infection and its relation to cigarette smoking.

\section{Methods}

Patients studied were those attending for upper digestive endoscopy on the routine and emergency

Correspondence: M.C. Bateson, M.D., F.R.C.P., F.R.C.P. (Edin.).

Accepted: 23 July 1992 lists held by a gastroenterologist in a district general hospital serving a population of 153,000 . Indications were varied including dyspepsia, heartburn, gastrointestinal bleeding, anaemia and biopsy of the small bowel. The local policy is as far as possible to avoid diagnostic endoscopy during or in the 2 months after a course of ulcer-healing therapy. None of the patients had ever received omeprazole or a prescription for bismuth salts. One patient in the validation study with active chronic duodenal ulcer was receiving ranitidine $300 \mathrm{mg}$ twice daily. No other patient had received ulcerhealing therapy with H2-receptor antagonists or other specific agents, nor antibiotics, for at least 8 weeks prior to attendance. The studies were designed prospectively and the only eligible patients who were not included were those few on anticoagulants or with clotting disorders, where biopsy would have been potentially hazardous. All patients were Caucasian of British origin.

\section{Validation study}

Ninety-eight consecutive patients were studied. Four other patients in the same period had to be excluded because they had had a partial gastrectomy. Diagnostic endoscopy was performed by a single operator and macroscopic findings noted. The largest available biopsy forceps were used to take three adjacent but not overlapping biopsies from the gastric antrum $20 \mathrm{~mm}$ from the pyloric rim.

1. One biopsy sample was immersed in brainheart infusion broth and transported immediately to the microbiology department for culture and smear for microscopy using Gram stain. All inoculations were performed within an hour of biopsy. Specimens were incubated for 5 days at $37^{\circ} \mathrm{C}$ in $10 \% \mathrm{CO}_{2}$ 
on Brucella medium with $8 \%$ lysed horse blood, and with vancomycin, colistin, amphotericin and trimethoprim added. Plates were examined for small translucent colonies. Confirmation of positive $H$. pylori culture was made by Gram stain of these colonies. Each culture preparation was accompanied by a control incubation of $H$. pylori. ${ }^{11}$

2. The second biopsy was immediately embedded in urea gel with phenol red indicator (CLO test) and kept at room temperature. The slide was read at 20 minutes, 3 hours and 16 hours. A positive result was a completely magenta urea gel cell, and a negative result was unchanged persistence of the initial yellow colour. All slides were read by the same investigator.

3. The third biopsy was placed in formol saline and sent for routine paraffin section using haemotoxylin and eosin staining. These were read by either of two consultant pathologists. The sections were separately stained with the full Giemsa technique prior to microscopy for H. pylori. ${ }^{11,12}$

\section{Smoking and peptic ulcer}

In a prospective study, consecutive endoscopy patients who had had either completely normal appearances or only a duodenal or gastric ulcer had a gastric antral biopsy embedded into a CLO test slide and interpreted as described above. Each patient was questioned about smoking habits at the time of endoscopy. They were classified as either current cigarette smokers, or as non-smokers if they had never used tobacco. Ex-smokers who had given up cigarettes at least a year before were classified with the non-smokers. Patients who smoked pipes or cigars were excluded from the study unless they were also current cigarette smokers.

Results were analysed by age, sex, smoking habit and endoscopic diagnosis. Where appropriate, statistical significance was examined by a $\chi^{2}$ test.

\section{Results}

\section{Validation study}

In nine out of the 98 patients control organisms did not grow on culture, so results could only be analysed in the other 89 . In the 43 culture-positive cases, 42 were also positive for CLO test (98\%); 34 and 36 , respectively, were positive on microscopy using Gram or Giemsa stains; and 28 had definite antral gastritis on histology. In the 46 culturenegative cases, 41 were also negative for CLO test $(89 \%) ; 38$ and 37 were negative on microscopy using Gram or Giemsa stains; and 27 had com- pletely normal histology, but another 11 had equivocal changes not diagnostic of chronic gast- $\frac{2}{3}$ ritis.

CLO test was at least as good as microscopy in $\frac{c}{.}$ detecting culture-positive and culture-negative $\Rightarrow$ cases, and correlated better with histological gas- $\stackrel{\rho}{\stackrel{\rho}{\rho}}$ tritis than any other test.

\section{H. pylori; smoking and peptic ulcer}

Of the positive CLO test results indicating $\frac{2}{\infty}$ Helicobacter pylori infection, $88.5 \%$ were identified $\overrightarrow{ }$ correctly by 20 minutes, $6.6 \%$ only changed by $3 \stackrel{\circ}{\circ}$ hours, and $4.9 \%$ were only apparent after 16 hours. $\vec{\omega}$ There was no detectable clinical difference between $\stackrel{\circ}{\circ}$ patients with early or later positive urease tests.

For patients with normal endoscopy the overall average ages were similar for those with positive 8 (52.2 years, $95 \%$ confidence interval $49.2-55.1 \%$ years), and negative results (50.3 years, $\mathrm{CI} \stackrel{\text { ㄱ. }}{ }$ 47.4-53.2), though the rate of infection was rather $\nexists$ higher in ages $50-69$ years than either younger or 0 older patients (Table I). There was no sex? difference, with $39.3 \%$ men and $40.6 \%$ women c having positive results.

Smokers were younger, with a mean age of $46.3 \stackrel{\frac{c}{2}}{2}$ years (CI 43.4-48.3) compared with 53.2 years $\left(\mathrm{CI}^{\rightleftharpoons}\right.$ 50.8-56.6) for non-smokers or ex-smokers. This $\vec{\ominus}$ might have been expected to produce a slighty lower infection rate in smokers. On the contrary tie striking new finding was a higher proportion of $\boldsymbol{H}$. pylori infection in current cigarette smokers $\left(73 / \frac{\partial}{2}\right.$ $147,49.6 \%$ ) compared with non-smokers plus ex-smokers $(112 / 315,35.5 \%, P<0.01)$. Similar $\stackrel{\circ}{\mathbb{Q}}$ differences were seen in men and women.

The strong association of $H$. pylori with peptic $\overrightarrow{\vec{O}}$ ulcer was confirmed. A total of 92 out of $108(85 \%)$ patients with duodenal ulcers were infected $(P<0.005)$ and 24 out of $43(55.8 \%)$ of patients with gastric ulcer $(P<0.01)$ compared with $185 \frac{0}{3}$ out of $462(40 \%)$ with normal endoscopy. In

Table I Age distribution of $H$. pylori infection by antral biopsy/urea gel/indicator test in patients with normal 윽 endoscopy

\begin{tabular}{|c|c|c|}
\hline Age (years) & $\begin{array}{c}\text { Proportion } \\
\text { positive }\end{array}$ & $\begin{array}{c}\text { Percentage } \\
\text { positive }\end{array}$ \\
\hline $14-19$ & $4 / 17$ & 23.5 \\
\hline $20-29$ & $20 / 63$ & 31.7 \\
\hline $30-39$ & $25 / 67$ & 37.3 \\
\hline $40-49$ & $28 / 78$ & 35.9 \\
\hline $50-59$ & $24 / 50$ & 48.0 \\
\hline $60-69$ & $43 / 67$ & 64.2 \\
\hline $70-79$ & $30 / 84$ & 35.7 \\
\hline $80-92$ & $11 / 46$ & 30.6 \\
\hline All ages & $185 / 462$ & 40.0 \\
\hline
\end{tabular}


addition the association of current cigarette smoking with peptic ulcer disease was confirmed. A total of 56 out of $108(51.8 \%)$ duodenal ulcer patients smoked $(P<0.001)$ and 21 out of $43(48.8 \%)$ gastric ulcer patients $(P<0.01)$ compared with 147 out of $462(31.8 \%)$ controls.

\section{Discussion}

The use of gastric antral urease testing with a urea gel/indicator system was shown to be sensitive and specific for current $H$. pylori infection. ${ }^{13,14}$ Actual culture of this fastidious organism is difficult despite great care and prolonged incubation, and a $10 \%$ failure rate has been demonstrated by others. ${ }^{15}$

It is agreed in the literature that there is no overall difference in $H$. pylori infection rates between men and women and this is consistent with the present study. The prevalence of infection in the community has been generally thought to increase with age,,$^{16,17}$ but the number of patients studied over 60 years has been much smaller than younger age groups. In Peru with a high carriage rate of $H$. pylori by direct testing there is no increase in infection rates with age in adults. ${ }^{18}$ There was also no age-related increase in positive IgG serology in Scotland, ${ }^{19}$ which has demographic and health characteristics similar to the north east of England.

The very strong association of $H$. pylori with duodenal ulcer and the strong association of $H$. pylori with gastric ulcer was confirmed, and is present after standardization for age in both sexes. Both types of peptic ulcer were significantly more frequent in smokers. There was also a strong correlation between active $H$. pylori infection itself and cigarette smoking in the group with normal endoscopy. This has previously been controver- sial. ${ }^{20,21}$ This series was rigorously prospective and all the endoscopic diagnoses and urease tests were made by a single observer. The patients with normal endoscopy were drawn from a stable community and probably represent the results which would be found by investigations of the rest of the population who are asymptomatic. Every subject was a caucasian of British origin, which avoided any bias of racial differences which might occur in mixed or migrant populations. ${ }^{20}$

Because of this homogenous study population, a sharply focused protocol and the elimination of observer variability, the present series is probably the best available evidence and establishes a definite association between current cigarette smoking and $H$. pylori infection. This may be explained by the effect cigarette smoking has on suppressing immunity. It is of considerable interest also because $H$. pylori has been incriminated in the aetiology of gastric cancer, which is also commoner in smokers. ${ }^{22,23}$

The results support the hypothesis that current cigarette smokers are predisposed to peptic ulcers because of increased susceptibility to $H$. pylori infection.

The urease urea gel/indicator assay system was CLO test manufactured by Delta West Limited, 15 Brodie Hall Drive, Bentley, Western Australia.

\section{Acknowledgement}

This study was only possible with the enthusiastic assistance of Dr F. Ahmad, consultant microbiologist, Drs K.A. Jasim and A. Senadhira, consultant histopathologists, and the staff of the Gastroenterology Unit at Bishop Auckland General Hospital.

\section{References}

1. Holt, P.G. Immune and inflammatory function in cigarette smokers. Thorax 1987, 42: 241-249.

2. Editorial. Smoking and immunity. Lancet 1990, 335: $1561-1563$.

3. Srivastava, E.D., Barton, J.R., O'Mahoney, S. et al. Smoking, humoral immunity and ulcerative colitis. Gut 1991, 32: 1016-1019.

4. Cope, G.F. \& Heatley, R.V. Cigarette smoking and intestinal defences. Gut 1992, 33: 721-723.

5. Kaufmann, D., Wilder-Smith, C.H., Kempf, M. et al. Cigarette smoking, gastric acidity and peptic ulceration. What are the relationships? Dig Dis Sci 1990, 35: 1482-1487.

6. Marshall, B. Unidentified curved bacillus on gastric epithelium in active chronic gastritis. Lancet 1983, 1: 1273-1275.

7. Dooley, C.P. \& Cohen, H. The clinical significance of Helicobacter pylori. Ann Intern Med 1988, 108: 70-79.

8. Graham, D.Y. Campylobacter pylori and peptic ulcer disease. Gastroenterology 1989, 96: 615-625.

9. Rauws, E.A.J. \& Tytgat, G.N.J. Cure of duodenal ulcer associated with eradication of Helicobacter pylori. Lancet 1990, 335: 1233-1235.

10. Editorial. Gastroenterologists in Sydney. Histology \& Helicobacter. Lancet 1990, 336: 779-780.

11. Gray, S.F., Wyatt, J.I. \& Rathbone, B.J. Simplified techniques for identifying Campylobacter pyloridis. J Clin Path 1986, 39: 1279.

12. Schnell, G.A. \& Schubert, T.T. Usefulness of culture, histology and urease testing in the detection of Campylobacter pylori. Am J Gastroenterol 1989, 84: 133-137.

13. Marshall, B.J., Warren, J.R., Francis, G.J., Langton, S.R., Goodwin, C.S. \& Blincow, E.D. Rapid urease test in the management of Campylobacter pyloris - associated gastritis. Am J Gastroenterol 1987, 82: 200-210.

14. Szet, M.L., Pounder, R.E., Hamilton-Dutoit, S.J. \& Dhillon, A.P. Rapid urease test provides specific identification of Campylobacter pylori in antral mucosal biopsies. Postgrad Med J 1988, 64: 935-936. 
15. Dooley, C.P., Cohen, H., Fitzgibbons, P.L. et al. Prevalence of Helicobacter pylori infection and histologic gastritis in asymptomatic persons. $N$ Engl J Med 1989, 321: 1562-1566.

16. Blaser, M.J. Epidemiology of Helicobacter pylori infection. In: Malfertheiner, P. \& Ditschunheit, H. (eds) Helicobacter pylori, Gastritis and Peptic Ulcer. Springer-Verlag, Berlin, 1990, pp. $38-40$.

17. Sitas, F., Forman, D., Yarnell, J.W.G. et al. Helicobacter pylori infection rates in relation to age and social class in a population of Welsh men. Gut 1991, 32: 25-28.

18. The Gastrointestinal Physiology Working Group. Helicobacter pylori and gastritis in Peruvian patients: relationship to socioeconomic level, age and sex. Am J Gastroenterol 1990, 85: 819-823.

19. Newell, D.G., Caygill, C.P.J., Stacey, A.R. \& Hill, M. The distribution of anti-C. pylori antibodies in patients undergoing endoscopy and in the normal population relative to age and geographical distribution. Proceedings of the 2nd Tokyo International Symposium on Campylobacter pylori, Tokyo, 1990, pp. 54-60.
20. Graham, D.Y., Malaty, H.M., Evans, D.G., Klein, P.D. \& Adam, E. Epidemiology of $\boldsymbol{H}$. pylori in an asymptomatic population in the US. Gastroenterology 1991, 100: 1495-1501.

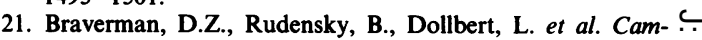
pylobacter pylori in Israel: prospective study of prevalence $\vec{F}$ and epidemiology. Israeli J Med Sci 1990, 26: 434-438.

22. Parsonnet, J., Friedman, G.D., Vandersteen, D.P. et al. Helicobacter pylori infection and the risk of gastric carcinoma. N Engl J Med 1991, 325: 1127-1131.

23. Nomura, A., Stemmermann, G.N., Chyou, P.-H., Kato, I., Perez-Perez, G.I. \& Blaser, M.J. Helicobacter pylori infection $\mathbb{\complement}$ and gastric carcinoma among Japanese Americans in Hawaii. N Engl J Med 1991, 325: 1132-1136. 